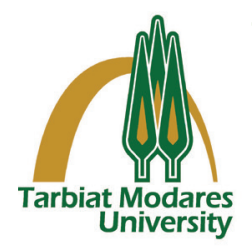

\title{
Geographical Distribution of COVID-19 Confirmed Cases in Iran: A Short Communication
}

\section{A R T I C L E I N F O}

\section{Article Type}

Short Communication

Authors

Moslem Taheri Soodejani, $P h D^{1}$ Hamid Reza Shoraka, $P h D^{2}$ Seyyed Mohammad Tabatabaei, $P h D^{3,4^{*}}$

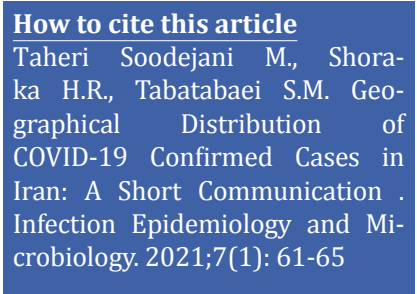

${ }^{1}$ Department of Epidemiology, School of Public Health, Shahid Sadoughi University of Medical Sciences, Yazd, IR, Iran

${ }^{2}$ Vector-Borne Diseases Research Center, North Khorasan University of Medical Sciences, Bojnurd, Iran ${ }^{3}$ Medical Informatics Department, School of Medicine, Mashhad University of Medical Sciences, Mashhad, Iran

${ }^{4}$ Department of fighting against Disease Najaf Abad Health Services center, Esfahan University of Medical Sciences, Najaf Abad, Iran

\section{* Correspondence}

Address: Department of fighting against Disease Najaf Abad Health Services center, Esfahan University of Medical Sciences, Najaf Abad, Iran. moha_taba@yahoo.com

\section{Article History}

Received: October 05,2020

Accepted: December 15

Published: January 23,2021

\section{A B S T R A C T}

Background: In Iran, the first cases of SARS-CoV-2 disease were detected with the death of 2 people in Qom city. Then other cases were reported in Markazi, Tehran, and Gilan provinces, and after that the disease spread to all 31 provinces of the country.

Materials and Methods: All data used in this study were collected from the reports of the National Committee on COVID-19 Epidemiology in the Ministry of Health and Medical Education in Iran. To investigate the effect of traveling between neighboring provinces, a spatial rate smoothing method was used, showing the impact of neighborhood on the disease prevalence. Also, to investigate the relationship between population density and disease prevalence, spatial regression was used at a significance level of $5 \%$.

Findings: Based on the estimated spatial rates, the disease prevalence rates changed in many provinces compared to the raw prevalence rates. Population density was also found to be directly related to the disease prevalence, so that with increasing population density, the disease prevalence rate increased $(p<.001)$.

Conclusion: It seems that case finding process should be done actively in all provinces of Iran regardless of administrative borders. Provinces should also be classified in terms of the disease transmission risk according to population density of patients, which may indicate the probability of contact between individuals.

\section{Keywords: SARS-COV-2, Coronavirus, Novel coronavirus, Spatial analysis.}

\section{CITATION LINKS}

[1] Adhikari SP, Meng S, Wu Y-J, Mao Y-P, Ye R-X, Wang Q-Z, et al. Epidemiology, causes, clinical manifestation and diagnosis, prevention, and control of coronavirus disease (COVID-19) during the early outbreak period: a scoping review. Infect Dis Poverty. 2020;9(1):1-12. [2] World Health Organization. WHO coronavirus disease (COVID-19) dashboard. Geneva: World Health Organization; 2020. [3] Taheri Soodejani M, Lotfi $\mathrm{MH}$, Tabatabaei SM. Is case fatality rate an appropriate index to represent the status of case-finding process for COVID-19 in different countries? Infect Ecol Epidemiol. 2020;10(1):1773733. [4] Arab-Mazar Z, Sah R, Rabaan AA, Dhama K, Rodriguez-Morales AJ. Mapping the incidence of the COVID-19 hotspot in Iran-Implications for Travellers. Travel Med Infect Dis. 2020. [5] Taheri Soodejani M, Tabatabaei SM, Dehghani A, McFarland W, Sharifi H. Impact of mass screening on the number of confirmed cases, recovered cases, and deaths due to COVID-19 in Iran: An Interrupted Time Series Analysis. Arch Iran Med. 2020;23(11):776-81. [6] MoHME. Corona statistics in IRAN. Ministry of Health and Medical Education(MoHME); 2020. [7] Goovaerts P. Geostatistical analysis of disease data: Estimation of cancer mortality risk from empirical frequencies using Poisson kriging. Int J Health Geogr. 2005;4(1):31. [8] Sohrabi C, Alsafi Z, O'Neill N, Khan M, Kerwan A, Al-Jabir A, et al. World Health Organization declares global emergency: A review of the 2019 novel coronavirus (COVID-19). Int J Surg. 2020. [9] Chinazzi M, Davis JT, Ajelli M, Gioannini C, Litvinova M, Merler S, et al. The effect of travel restrictions on the spread of the 2019 novel coronavirus (COVID-19) outbreak. Science. 2020; 368(6489): 395-400. [10] Liu Y, Gayle AA, Wilder-Smith A, Rocklöv J. The reproductive number of COVID-19 is higher compared to SARS coronavirus. J Travel Med. 2020. [11] Rocklöv J, Sjödin H. High population densities catalyze the spread of COVID-19. 


\section{Introduction}

In December 2019, a viral disease with respiratory symptoms was identified in Wuhan, China, and spread rapidly to other parts of China and the world. Currently, almost all countries in the world are infected with this virus, and it is considered as the most important challenge to the global health as it has spread out to 210 countries around the world ${ }^{[1-2]}$.

Concerning the new coronavirus characteristics, most of people infected have no symptom or mild symptom. Thus, many cases are not detected, and this situation causes to underestimate the disease prevalence rate in all countries ${ }^{[3]}$.

In Iran, the first cases of SARS-CoV-2 disease were detected with the death of 2 people in Qom city. Then other cases were reported in Markazi, Tehran, and Gilan provinces, and after that it spread to all 31 provinces of the country. However, the pattern of the disease prevalence in the provinces of the country has not been the same, so that the prevalence rate of the disease in some provinces has been higher than in other provinces, most of which have been neighboring provinces of Qom and Tehran ${ }^{[4]}$. Iran is one of the most severely affected countries, with the highest number of reported infected cases and deaths in the Mediterranean Region ${ }^{[5]}$.

Objectives: Since traveling to neighboring provinces may cause the disease to spread out more and more, the aim of this study was to investigate the geographical distribution of the disease as well as the effect of geographical location and population density on the prevalence of SARS-CoV-2 disease.

\section{Materials and Methods}

Data Access: All data used in this study were collected from the reports of the National Committee on COVID-19 Epidemiology in the Ministry of Health and Medical Education (MOHME) in Iran. Infected cases wereidentified according to SARS-CoV-2 diagnosis and treatment guidelines published by MOHME [6]. Statistical analysis: The disease raw prevalence in each province was calculated by dividing the number of confirmed cases by the population of each province and presented using ARCGIS Version 10 with colored ranges (based on quarters). Considering the interprovincial travels, it was possible that the disease prevalence in each province would be almost the same as in neighboring provinces. To investigate this hypothesis, the Spatial Rate smoothing method was used. In this method, smoothing is done by considering the location of each province and the disease prevalence in its neighboring provinces, then according to the weighting type used, the prevalence is smoothed. Quinn model was used for weighting, which included all neighboring provinces. In this method, provinces with even the least common borders are considered as neighbors. After finding the neighbors, the average weight of the neighboring provinces is used to smooth out the disease prevalence in each province ${ }^{[7]}$.

Also, to compare the probable prevalence of the disease in all provinces, the probable prevalence rate in each one was calculated based on population density. Population density was calculated through dividing the population of each province by its area. Spatial regression was used at a significance level of $5 \%$ to investigate the relationship between population density and the disease prevalence.

\section{Findings}

Based on the reported results of confirmed cases in Iran by MOHME, until March 23, 2020 (before the start of mass screening), the lowest prevalence rate was observed in the provinces of Kermanshah, Chaharmahal and Bakhtiari, Khuzestan, Bushehr, Fars, Kerman, Hormozgan, and Sistan and Baluchestan, and the highest prevalence rate was observed in the provinces of Mazandaran, Qom, Gilan, Semnan, Qazvin, Markazi, and Yazd (Figure 1). 


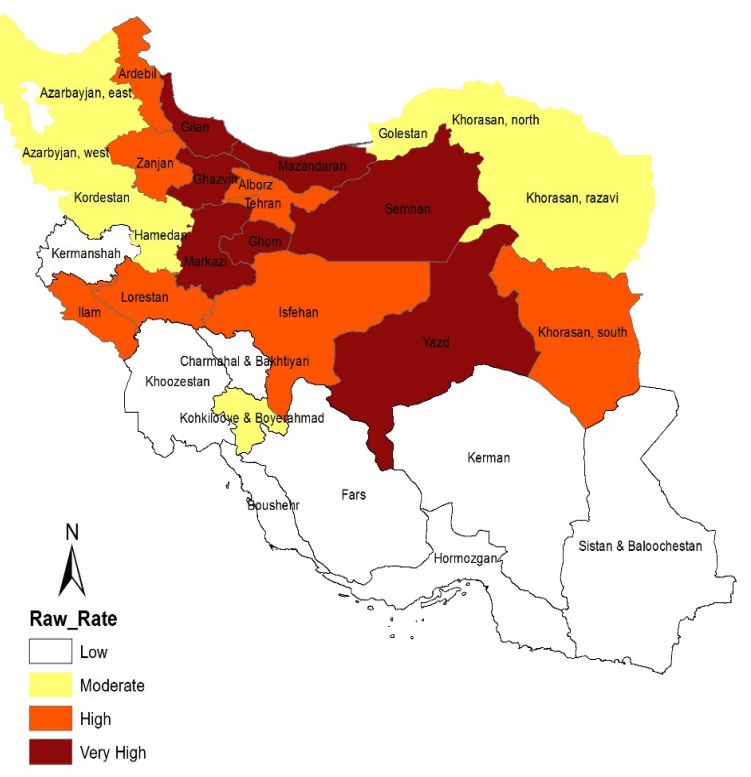

Figure 1) Distribution of COVID-19 prevalence in Iran based on the reported results of confirmed cases

The spatial rate estimation results showed that the provinces of Kurdistan, Ilam, Khuzestan, Kohgiluyeh and Boyer-Ahmad, Bushehr, Hormozgan, Kerman, and South Khorasan had the lowest prevalence rate. On the other hand, the provinces of Tehran, Golestan, Mazandaran, Gilan, Qazvin, Qom, and Sistan and Baluchestan had the highest prevalence rate (Figure 2).

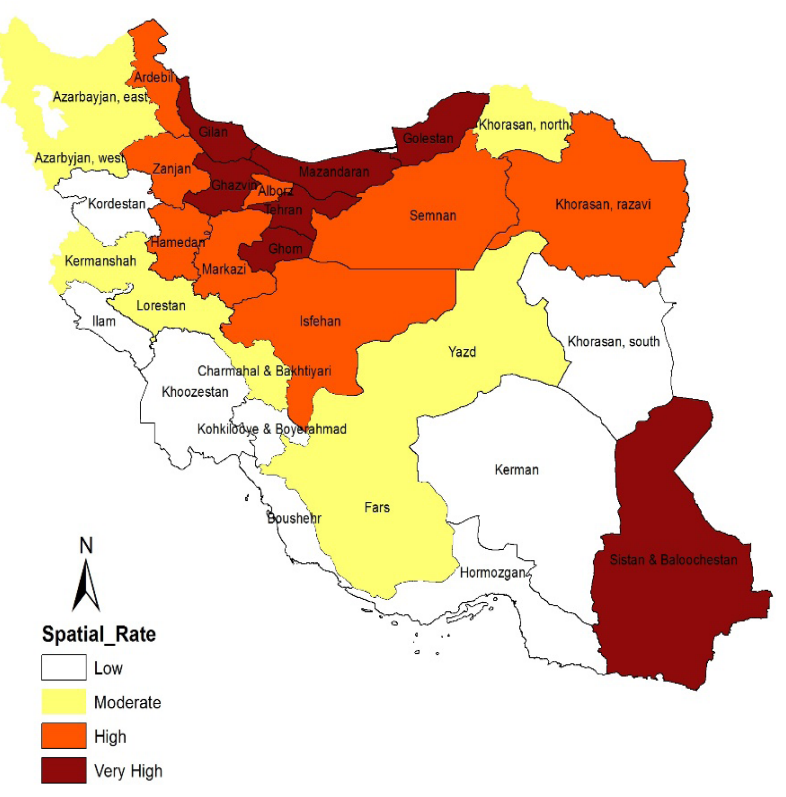

Figure 2) Distribution of COVID19- prevalence in Iran based on spatial rate estimation
Investigating the relationship between population density and disease prevalence showed that there is a significant relationship between the two $(p<.001)$, so that with increasing the population density, the disease prevalence also increased. After applying the effect of inter-provincial communication (Spatial Rate) and also population density on the disease prevalence, it was found that the provinces including Alborz, Tehran, East Azerbaijan, West Azerbaijan, Gilan, Kermanshah, Khuzestan, and Bushehr had the lowest number of infected cases, while North Khorasan provinces. Semnan, Isfahan, Markazi, Yazd, South Khorasan, and Sistan and Baluchestan had the highest number of infected cases based on population density (Figure 3).

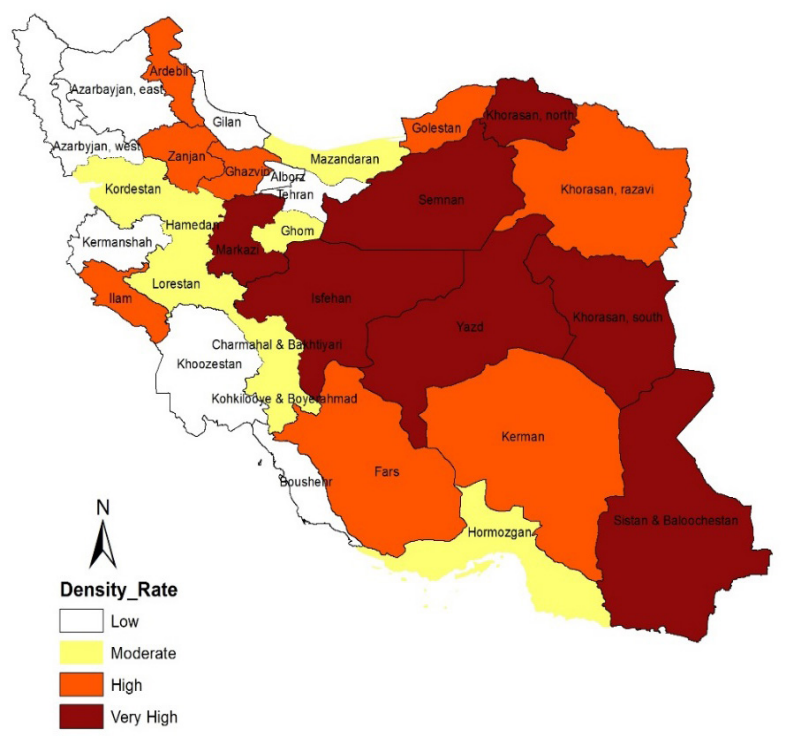

Figure 3) Classification of high-risk locations based on population density in different provinces of Iran

\section{Discussion}

Based on the reported results of confirmed cases, the prevalence of this disease in some provinces is lower than in other provinces, while in their neighborhood, there are provinces with a very high prevalence of the disease. According to the inter-provincial communications, administrative borders could not properly represent the disease distribution in each province, and it is recommended to 
somehow adjust them.

Inter-provincial travels and communications may cause faster transmission of the disease between provinces ${ }^{[8-9]}$ if neighboring provinces have the highest prevalence rate of the disease. According to the pattern used for the interprovincial communications to estimate the Spatial Rate, it appears that inter-provincial communications have led to faster transmission of the disease. It seems that provinces such as Chaharmahal and Bakhtiari, Sistan and Baluchestan, and Fars, which are apparently at a low-risk level, according to the reports, may need a better case-finding process and more reports. It seems that the provinces such as Yazd, Semnan, Mazandaran, Gilan, Tehran, Qazvin, and Qom have performed better in case-finding process than other provinces. Another factor that has a high impact on the transmission of this disease is the contact rate between individuals, which is one of the parameters in calculating Reproduction Number Rate (R0) ${ }^{[10]}$. The population density of each province may indicate the probability of contact between individuals ${ }^{[11]}$. Based on this and also according to the inter-provincial communications (Spatial Rate), a different distribution of the disease prevalence in Iran was obtained. The provinces of Tehran, Alborz, and Gilan, which are among the highprevalence provinces, seems to be at a lowrisk level when applying the number of cases predicted based on population density. This indicates that population density in different provinces in Iran is one of the important factors in determining the risk of infection.

\section{Study restrictions}

Factors such as the status of case-finding as well as the existing traditions in each province may increase or decrease the number of infected cases among them. In this study, no such data were available.

\section{Conclusion}

It seems that the case-finding process should be done actively in all provinces in Iran, regardless of administrative borders. Also, to classify provinces in terms of the disease transmission risk, the population density, which may represent the contact rate among individuals, should be used.

Acknowledgments: We would like to thank all staff of Ministry of Health and Medical Education.

Ethical Permission: Not applicable.

Authors Contribution: Conceptualization: MTS; Data curation and formal analysis: MTS and HR; Investigation: MTS and SMT; Methodology and project administration: MTS; Supervision: SMT; Validation: All authors; Writing of original draft: MTS; Writing, reviewing, and editing: SMT.

Conflict of interest: None.

Fundings: This study had no financial support.

Consent to participate: Not applicable.

\section{References}

1. Adhikari SP, Meng S, Wu Y-J, Mao Y-P, Ye R-X, Wang Q-Z, et al. Epidemiology, causes, clinical manifestation and diagnosis, prevention, and control of coronavirus disease (COVID-19) during the early outbreak period: a scoping review. Infect Dis Poverty. 2020;9(1):112.

2. World Health Organization. WHO coronavirus disease (COVID-19) dashboard. Geneva: World Health Organization; 2020.

3. Taheri Soodejani M, Lotfi MH, Tabatabaei SM. Is case fatality rate an appropriate index to represent the status of casefinding process for COVID-19 in different countries? Infect Ecol Epidemiol. 2020;10(1):1773733.

4. Arab-Mazar Z, Sah R, Rabaan AA, Dhama $\mathrm{K}$, Rodriguez-Morales AJ. Mapping the incidence of the COVID-19 hotspot in Iran-Implications for Travellers. Travel 
Med Infect Dis. 2020.

5. Taheri Soodejani M, Tabatabaei SM, Dehghani A, McFarland W, Sharifi H. Impact of mass screening on the number of confirmed cases, recovered cases, and deaths due to COVID-19 in Iran: An Interrupted Time Series Analysis. Arch Iran Med. 2020;23(11):776-81.

6. MoHME. Corona statistics in IRAN. Ministry of Health and Medical Education(MoHME); 2020.

7. Goovaerts P. Geostatistical analysis of disease data: Estimation of cancer mortality risk from empirical frequencies using Poisson kriging. Int J Health Geogr. 2005;4(1):31.

8. Sohrabi C, Alsafi Z, O'Neill N, Khan M,
Kerwan A, Al-Jabir A, et al. World Health Organization declares global emergency: A review of the 2019 novel coronavirus (COVID-19). Int J Surg. 2020.

9. Chinazzi M, Davis JT, Ajelli M, Gioannini C, Litvinova M, Merler S, et al. The effect of travel restrictions on the spread of the 2019 novel coronavirus (COVID-19) outbreak. Science. 2020; 368(6489):395400.

10. Liu Y, Gayle AA, Wilder-Smith A, Rocklöv J. The reproductive number of COVID-19 is higher compared to SARS coronavirus. J Travel Med. 2020.

11. Rocklöv J, Sjödin H. High population densities catalyze the spread ofCOVID-19. J Travel Med. 2020;27(3):taaa038. 